\title{
Spectral properties of supersymmetric Polychronakos spin chain associated with $A_{N-1}$ root system
}

\author{
B. Basu-Mallich* and Nilanjan Bondyopadhayat \\ Theory Group, \\ Saha Institute of Nuclear Physics, \\ 1/AF Bidhan Nagar, Kolkata 700 064, India
}

\begin{abstract}
By using the exact partition function of $s u(m \mid n)$ Polychronakos spin chain associated with $A_{N-1}$ root system, we study some statistical properties of the related spectrum. It is found that the corresponding energy level density satisfies the Gaussian distribution and the cumulative distribution of spacing between consecutive energy levels obeys a certain 'square root of a logarithm' law.

PACS: 02.30.Ik; 75.10.Jm; 05.30.-d; 75.10.Pq

Keywords: Exactly solvable quantum spin chains; Supersymmetry; Partition function; Level density distribution
\end{abstract}

* e-mail address: bireswar.basumallick@saha.ac.in

$\dagger$ e-mail address: nilanjan.bondyopadhaya@saha.ac.in 
Exactly solvable one dimensional quantum integrable spin chains and dynamical models with long-range interaction [1-11] have attracted much attention in recent years due to their appearance in a wide range of subjects like fractional statistics [12, 13], quantum electric transport in mesoscopic systems [14], Yangian quantum group [15-17], SUSY Yang-Mills theory and string theory [18-20]. Among quantum spin systems with longrange interaction, the well known spin- $\frac{1}{2}$ Haldane-Shastry (HS) model is introduced in an attempt to construct an exact ground state which would coincide with the $U \rightarrow \infty$ limit of Gutwiller's variational wave function for the Hubbard model [4, 5]. A natural $s u(m)$ generalization of this exactly solvable HS model is constructed by using the 'spin' exchange operator associated with the fundamental representation of $s u(m)$ algebra [6, 7]. Subsequently, it is realized that such HS spin chain may be reproduced from the trigonometric spin Calogero-Sutherland model by applying the 'freezing trick', which basically uses the fact that spin and dynamical degrees of freedom of the latter model decouple from each other for large values of the coupling constant. Furthermore, a new quantum spin chain with long-range interaction is obtained by applying this freezing trick to the case of rational spin Calogero model [8]. Lattice sites of this spin chain, which are inhomogeneously distributed on a line, are determined through the zeros of the Hermite polynomial [10]. This quantum integrable as well as exactly solvable spin system is usually known as Polychronakos or Polychronakos-Frahm spin chain in the literature. Both HS and Polychronakos spin chains admit $s u(m \mid n)$ supersymmetric extensions, in which each site is occupied by either one of the $m$ type of bosonic states or one of the $n$ type of fermionic states [21-27]. Such supersymmetric spin chains play a role in describing some strongly correlated systems in condensed matter physics, where holes moving in the dynamical back ground of spins behave as bosons, and spin- $1 / 2$ electrons behave as fermions [28, 29].

It should be noted that, the above mentioned HS and Polychronakos spin chains (along with their supersymmetric extensions) are all related to the $A_{N-1}$ type of root system, for 
which the interaction between any two spins depends only on the difference of their site coordinates. Variants of these spin chains associated with other root systems, endowed with more general type of interactions, have also been studied in the literature [30-36].

By applying the method of freezing trick, it has become possible to compute the exact partition functions of $s u(m)$ Polychronakos and $s u(m)$ HS spin chains associated with $A_{N-1}$ root system [9, 37], $s u(m \mid n)$ supersymmetric extensions of these spin chains [23, 25], and variants of such spin chains related to other root systems [33-36]. These exact partition functions have turned out to be a very efficient tool for studying some statistical properties of the related energy spectra like level density distribution and distribution of spacing between consecutive energy levels. It is found that, for sufficiently large number of lattice sites, the energy level density of this type of spin chain follows the Gaussian distribution with high degree of accuracy [33-38,25]. It is also observed that, distribution of spacing between consecutive energy levels for such integrable spin chains is not of Poisson type, as may be expected due to a well-known conjecture of Berry and Tabor [39]. However it appears that, even though the exact partition function of $s u(m \mid n)$ supersymmetric Polychronakos spin chain associated with $A_{N-1}$ root system has been derived by using the freezing trick [23], statistical properties of the related spectrum like distributions of energy level density and spacing between consecutive energy levels have not been analyzed till now. The purpose of this letter is to study these spectral properties of the supersymmetric Polychronakos spin chain by using its exact partition function.

The Hamiltonian of the $s u(m \mid n)$ supersymmetric Polychronakos spin chain associated with the $A_{N-1}$ root system is given by [23]

$$
\mathcal{H}^{(m \mid n)}=\sum_{1 \leq j<k \leq N} \frac{\left(1-\hat{P}_{j k}^{(m \mid n)}\right)}{\left(x_{j}-x_{k}\right)^{2}},
$$

where $x_{j}$ 's are the zeros of the $N$-th order Hermite polynomial, and the supersymmetric 
exchange operator $\hat{P}_{j k}^{(m \mid n)}$ is defined as

$$
\hat{P}_{j k}^{(m \mid n)} \equiv \sum_{\alpha, \beta=1}^{m+n} C_{j, \alpha}^{\dagger} C_{k, \beta}^{\dagger} C_{j, \beta} C_{k, \alpha}
$$

Here creation-annihilation operators like $C_{j, \alpha}^{\dagger}$ and $C_{j, \alpha}$ are bosonic for $\alpha \in\{1,2, \ldots, m\}$ and fermionic for $\alpha \in\{m+1, m+2, \ldots, m+n\}$, and these operators act on a restricted Hilbert space satisfying the constraint:

$$
\sum_{\alpha=1}^{m+n} C_{j, \alpha}^{\dagger} C_{j, \alpha}=1
$$

for all $j$. It may be noted that for the special case like $m \neq 0, n=0$ or $m=0, n \neq 0$, i.e. when all degrees of freedom are either bosonic or fermionic, $\mathcal{H}^{(m \mid n)}$ in Eq. (11) reduces to the non-supersymmetric Polychronakos spin chain, for which statistical properties of the spectrum have been studied very recently [38]. In this article we shall analyze the spectrum of $\mathcal{H}^{(m \mid n)}$ in the purely supersymmetric case, when both $m$ and $n$ take nonzero values.

As shown in Ref. [22, the supersymmetric exchange operator (2) can be mapped to an 'anyon like' representation of the permutation algebra on an appropriate spin space. By using this mapping and subsequently applying the freezing trick, the exact partition function of $s u(m \mid n)$ Polychronakos spin chain has been derived as 23

$$
Z_{N}^{(m \mid n)}(q)=\sum_{\sum_{i=1}^{m} a_{i}+\sum_{j=1}^{n} b_{j}=N} \frac{(q)_{N} \cdot q^{\sum_{j=1}^{n} \frac{b_{j}\left(b_{j}-1\right)}{2}}}{(q)_{a_{1}}(q)_{a_{2}} \cdots(q)_{a_{m}} \cdot(q)_{b_{1}}(q)_{b_{2}} \cdots(q)_{b_{n}}}
$$

where $a_{i}$ 's and $b_{i}$ 's are non-negative integers, $q \equiv e^{-1 / k T}$ and the notation: $(q)_{N} \equiv$ $(1-q)\left(1-q^{2}\right) \cdots\left(1-q^{N}\right) \quad\left(\right.$ with $\left.(q)_{0} \equiv 1\right)$ is used. Exploiting a connection between this partition function and supersymmetric Schur polynomials, it is found that all energy eigenvalues of $s u(m \mid n)$ Polychronakos spin chain can be expressed in terms of 'motifs', 
which are represented by arrays of binary digits like '0' and '1' [24]. For a spin chain with $N$ number of lattice sites, each motif is represented by an array of $N-1$ number of binary digits. Thus we may write such a motif as $\left(\delta_{1}, \delta_{2}, \ldots, \delta_{N-1}\right)$, where $\delta_{i} \in[0,1]$. These motifs characterize a class of irreducible representations of $Y(g l(m \mid n))$ Yangain quantum group, which span the Fock space of $s u(m \mid n)$ supersymmetric Polychronakos spin chain. Since $Y(g l(m \mid n))$ Yangain is the symmetry algebra of this spin chain, all of its eigenstates associated with any particular irreducible representation or corresponding motif yield the same energy eigenvalue. One can express the energy eigenvalue of such degenerate eigenfunctions associated with the motif $\delta \equiv\left(\delta_{1}, \delta_{2}, \ldots, \delta_{N-1}\right)$ as

$$
E(\delta)=\sum_{j=1}^{N-1} j \delta_{j} .
$$

It should be noted that, in contrast to the non-supersymmetric case, there is no restriction in arranging 1's or 0's in a motif associated with the supersymmetric Polychronakos spin chain [24]. Consequently, some additional motifs and energy levels appear in the spectrum of this supersymmetric spin chain in comparison with its non-supersymmetric counterpart. In fact, for this supersymmetric spin chain with $N$ number of lattice sites, one can construct $2^{N-1}$ number of motifs by filling up $N-1$ positions arbitrarily with either 0 or 1 . By using Eq. (4), it is easy to check that the motif $(0,0, \ldots, 0)$ yields the ground state with zero energy and the motif $(1,1, \ldots, 1)$ yields the highest excited state with energy $N(N-1) / 2$. Moreover, depending on the choice of $\delta, E(\delta)$ in Eq. (4) can take any integer value within the range 0 to $N(N-1) / 2$. Consequently, the spectrum of $s u(m \mid n)$ supersymmetric Polychronakos spin chain would be equispaced in nature.

Since $Z_{N}^{(m \mid n)}(q)$ in Eq. (3) represents the partition function of a finite spin system with integer eigenvalues, it should be expressed as a polynomial function of $q$. Indeed, with the help of a symbolic software package like Mathematica, we can explicitly write down $Z_{N}^{(m \mid n)}(q)$ as a polynomial of $q$ for wide range of values of $m, n$ and $N$. If the 
term $q^{E_{i}}$ appears in such a polynomial, then $E_{i}$ will represent an energy eigenvalue and the coefficient of $q^{E_{i}}$ will determine the degeneracy factor corresponding to this energy level. Let us denote this degeneracy factor or 'level density' associated with the energy level $E_{i}$ as $D^{(m \mid n)}\left(E_{i}\right)$. Since the sum of these level densities for the full spectrum is not normalized to unity, we define normalized level density as

$$
\mathcal{D}^{(m \mid n)}\left(E_{i}\right)=\frac{1}{(m+n)^{N}} D^{(m \mid n)}\left(E_{i}\right)
$$

such that $\sum_{E_{i}} \mathcal{D}^{(m \mid n)}\left(E_{i}\right)=1$. In the following, we shall use this $\mathcal{D}^{(m \mid n)}\left(E_{i}\right)$ to study some spectral properties of the supersymmetric Polychronakos spin chain.

Let us investigate at first whether, in analogy with the case of many other quantum integrable spin chains with long-range interaction, the energy level density of $s u(m \mid n)$ supersymmetric Polychronakos spin chain can be described by the Gaussian distribution. The form of normalized Gaussian distribution is given by

$$
G(E)=\frac{1}{\sqrt{2 \pi} \sigma} \exp \left[-\frac{(E-\mu)^{2}}{2 \sigma^{2}}\right]
$$

where $\mu$ and $\sigma$ denote the mean value and standard deviation respectively. For the case of supersymmetric Polychronakos spin chain, these parameters are evidently related to the Hamiltonian $\mathcal{H}^{(m \mid n)}$ in Eq. (11) as

$$
\mu=\frac{\operatorname{tr}\left[\mathcal{H}^{(m \mid n)}\right]}{(m+n)^{N}}, \quad \sigma^{2}=\frac{\operatorname{tr}\left[\left(\mathcal{H}^{(m \mid n)}\right)^{2}\right]}{(m+n)^{N}}-\mu^{2}
$$

In order to compare $\mathcal{D}^{(m \mid n)}\left(E_{i}\right)$ with $G\left(E_{i}\right)$, we have to compute the parameters $\mu$ and $\sigma$ in terms of $m, n$ and $N$. For this purpose, let us consider some trace formulas involving 
supersymmetric exchange operators like [25]

$$
\begin{aligned}
& \operatorname{tr}\left[\left(\hat{P}_{i j}^{(m \mid n)}\right)^{2}\right]=\operatorname{tr}[\mathbb{1}]=s^{N}, \quad \operatorname{tr}\left[\hat{P}_{i j}^{(m \mid n)}\right]=s^{N-2} t, \\
& \operatorname{tr}\left[\hat{P}_{i j}^{(m \mid n)} \hat{P}_{i l}^{(m \mid n)}\right]=\operatorname{tr}\left[\hat{P}_{i j}^{(m \mid n)} \hat{P}_{j l}^{(m \mid n)}\right]=\operatorname{tr}\left[\hat{P}_{i j}^{(m \mid n)} \hat{P}_{k j}^{(m \mid n)}\right]=s^{N-2}, \\
& \operatorname{tr}\left[\hat{P}_{i j}^{(m \mid n)} \hat{P}_{k l}^{(m \mid n)}\right]=s^{N-4} t^{2},
\end{aligned}
$$

where $s=m+n, t=m-n$ and $i, j, k, l$ are all different indices. With the help of these trace formulas, $\mu$ and $\sigma$ in Eq. (7) can be expressed as

$$
\begin{aligned}
\mu & =\frac{1}{2 s^{2}}\left(s^{2}-t\right) \sum_{\substack{i, j=1 \\
i \neq j)}}^{N} \frac{1}{x_{i j}^{2}}, \\
\sigma^{2} & =\frac{s^{4}-t^{2}}{2 s^{4}} \sum_{\substack{i, j=1 \\
i \neq j)}}^{N} \frac{1}{x_{i j}^{4}}+\frac{s^{2}-t^{2}}{s^{4}} \sum_{\substack{i, j, k=1 \\
i \neq j \neq k \neq i)}}^{N} \frac{1}{x_{i j}^{2} x_{j k}^{2}},
\end{aligned}
$$

where $x_{i j} \equiv x_{i}-x_{j}$. These two expressions encompass different types of summations involving the zeros of the $N$-th order Hermite polynomial. It is well known that, the zeros of the $N$-th order Hermite polynomial satisfy an identity of the form [40]

$$
\sum_{\substack{k=1 \\ k \neq i)}}^{N} \frac{1}{x_{i k}}=x_{i}
$$

Starting from this simple identity, it is possible to derive many other more complicated form of identities involving the zeros of the Hermite polynomial. In particular, one can show that 40]

$$
\sum_{\substack{i, j=1 \\ i \neq j)}}^{N} \frac{1}{x_{i j}^{2}}=\frac{1}{2} N(N-1), \quad \sum_{\substack{i, j=1 \\ i \neq j)}}^{N} \frac{1}{x_{i j}^{4}}=\frac{1}{36} N(N-1)(2 N+5) .
$$


By using the identity (11), we also find that

$$
\sum_{\substack{i, j, k=1 \\ i \neq j \neq k \neq i)}}^{N} \frac{1}{x_{i j}^{2} x_{j k}^{2}}=\frac{2}{9} N(N-1)(N-2)
$$

Substituting the identities given in Eqs. (12) and (13) to Eq. (10), we finally obtain $\mu$ and $\sigma$ in terms of $m, n$ and $N$ as

$$
\begin{aligned}
\mu & =\frac{1}{4 s^{2}}\left(s^{2}-t\right) N(N-1) \\
\sigma^{2} & =\frac{s^{4}-t^{2}}{72 s^{4}} N(N-1)(2 N+5)+\frac{2\left(s^{2}-t^{2}\right)}{9 s^{4}} N(N-1)(N-2) .
\end{aligned}
$$

From the above expression it is evident that, $\mu$ and $\sigma$ are of the order of $N^{2}$ and $N^{3 / 2}$ respectively at $N \rightarrow \infty$ limit. Interestingly, similar type of large $N$ behaviour of these parameters have also been observed for non-supersymmetric Polychronakos spin chain associated with the $A_{N-1}$ root system [38] and non-supersymmetric as well as supersymmetric Polychronakos spin chains associated with the $B C_{N}$ root system [34, 35].

Now we are in a position to estimate the agreement between $\mathcal{D}^{(m \mid n)}\left(E_{i}\right)$ and $G\left(E_{i}\right)$ for the case of $s u(m \mid n)$ supersymmetric Polychronakos spin chain. As an example, let us consider the simplest case of $s u(1 \mid 1)$ Polychronakos spin. By using Mathematica, we can easily express the corresponding partition function (given in Eq. (3) with $m=n=1$ ) as a polynomial of $q$ up to $N \approx 50$ and obtain $\mathcal{D}^{(1 \mid 1)}\left(E_{i}\right)$ for all values of $E_{i}$. By using such data for the specific case of $N=30$ lattice sites, we plot $\mathcal{D}^{(1 \mid 1)}\left(E_{i}\right)$ versus $E_{i}$ through the dotted curve in Fig. 1. Next, by substituting $N=30$ and $m=n=1$ in Eq. (14), we get $\mu=217.5$ and $\sigma=46.25$. The continuous curve in Fig. 1 plots $G(E)$ as a function of $E$ for these values of $\mu$ and $\sigma$. Comparison between the continuous and dotted curves in Fig. 1 confirms that the normalized energy level density obeys Gaussian distribution with a very high degree of accuracy. We also calculate the mean square error (MSE) for this case and find it as low as $4.74 \times 10^{-4}$. Moreover, it is found that this MSE decreases 
gradually with the increase of the number of lattice sites. We have compared $\mathcal{D}^{(m \mid n)}\left(E_{i}\right)$ with $G\left(E_{i}\right)$ for many other values of $m, n$ and $N$, and always found excellent agreement between them for sufficiently large number of lattice sites.

Next, our aim is to study the distribution of spacing between consecutive energy levels for the case of supersymmetric Polychronakos spin chain. To this end, we define cumulative level spacing distribution as

$$
P(s)=\int_{o}^{s} p(x) d x
$$

where $p(x)$ is the probability density of the normalized spacing $x$ between consecutive (unfolded) energy levels of the Hamiltonian. Unfolded energy levels are obtained by exploiting a mapping which smooths out the 'raw' spectrum containing local fluctuations [41. To define such unfolding mapping, at first it is needed to decompose the cumulative energy level density as the sum of a fluctuating part and a continuous part. This continuous part of the cumulative energy level density (denoted by $\eta(E)$ ) is then used to transform each energy $E_{i}, i=1,2, \ldots, l$, into an unfolded energy $\mathcal{E}_{i}: E_{i} \rightarrow \mathcal{E}_{i}=\eta\left(E_{i}\right)$. Since we have found that, the energy level density of $s u(m \mid n)$ Polychronakos spin chain follows the continuous Gaussian distribution with very good approximation, $\eta(E)$ can be expressed through the error function as

$$
\eta(E)=\int_{-\infty}^{E} G\left(E^{\prime}\right) d E^{\prime}=\frac{1}{2}\left[1+\operatorname{erf}\left(\frac{E-\mu}{\sqrt{ } 2 \sigma}\right)\right]
$$

Finally, the function $p\left(s_{i}\right)$ is defined as the density of normalized spacing $s_{i}=\left(\mathcal{E}_{i+1}-\right.$ $\left.\mathcal{E}_{i}\right) / \Delta$, where $\Delta=\left(\mathcal{E}_{l}-\mathcal{E}_{1}\right) /(l-1)$ is the mean spacing of the unfolded energy levels.

We have already mentioned that, according to a well-known conjecture of Berry and Tabor, the density $p(s)$ of normalized spacing for the case of a quantum integrable system should obey the Poisson's law: $p(s)=e^{-s}$ [39]. However, some recent works reveal that a class of quantum integrable HS and Polychronakos like spin chains exhibit non-Poissonian 
distribution of spacing between consecutive energy levels and thus violate the Berry-Tabor conjecture [33-38,25]. It is also found that, for the above mentioned type of spin chains, $P(s)$ obeys a certain 'square root of a logarithm' law, which can be derived analytically by assuming a few simple properties of the related spectra. More precisely, if any spectrum $E_{\text {min }} \equiv E_{1}<\cdots<E_{l} \equiv E_{\max }$ obeys the following features:

(i) the energy levels are equispaced, or, equivalently $E_{i+1}-E_{i}=\lambda$ for $1 \leq i<l$,

(ii) the normalized energy level density is approximately given by the Gaussian distribution,

(iii) two tails of the level density distribution are well spread, i.e, $E_{\max }-\mu, \mu-E_{\min }>>\sigma$,

(iv) $E_{\min }$ and $E_{\max }$ are approximately symmetric with respect to the mean energy $\mu$, namely $\left|E_{\max }+E_{\min }-2 \mu\right|<<E_{\max }-E_{\min }$,

then $P(s)$ is approximately given by an analytic expression of the form [34

$$
\bar{P}(s)=1-\frac{2}{\sqrt{\pi} s_{\max }} \sqrt{\log \left(\frac{s_{\max }}{s}\right)},
$$

where $s_{\max }$ denotes the maximum normalized spacing, which can be estimated with great accuracy through the relation

$$
s_{\max }=\frac{E_{\max }-E_{\min }}{\sqrt{2 \pi} \sigma} .
$$

Studying the case of $s u(m \mid n)$ supersymmetric Polychronakos spin chain for a wide range of values of $m, n$ and $N$, we also find that the spacing between consecutive energy levels does not follow the Poisson distribution. So it is natural to explore the applicability of expression (16) for the present case. To this end, let us first check whether the energy spectrum of $s u(m \mid n)$ supersymmetric Polychronakos spin chain satisfies the four conditions that have been described in the previous paragraph. We have already found that 
this spectrum is equally spaced with unit interval and the level density follows Gaussian distribution with good approximation for sufficiently large values of $N$. So this spectrum evidently satisfies the first two conditions. From Eq. (14) and the expressions of lowest and highest energy levels given by $E_{\min }=0$ and $E_{\max }=N(N-1) / 2$ respectively, it follows that both $\left(\mu-E_{\min }\right) / \sigma$ and $\left(E_{\max }-\mu\right) / \sigma$ vary as $\sqrt{N}$ when $N \rightarrow \infty$. Therefore, this spectrum conforms to the third condition. At $N \rightarrow \infty$ limit, it is also easy to find that

$$
\left|E_{\min }+E_{\max }-2 \mu\right|=X(m, n)\left(E_{\max }-E_{\min }\right)
$$

where $X(m, n)=\frac{|m-n|}{(m+n)^{2}}$. Note that $X(m, n)$ becomes zero for $m=n$ and takes a finite nonzero value for $m \neq n$. Consequently, the fourth condition is satisfied only for the case $m=n$. However it can be shown that, if one drops this forth condition, Eq. (16) still holds within a slightly smaller range of $s$ [35]. Therefore, it is natural to expect that $P(s)$ would follow the analytical expression $\bar{P}(s)(16)$ in the case $s u(m \mid n)$ Polychronakos spin chain for all possible values of $m, n$ and sufficiently large values of $N$.

To support the above conclusion, we study numerically the nature of $P(s)$ with different values of $m, n$ and $N$. For example, we may consider the particular case of $s u(1 \mid 1)$ spin chain with $N=30$ lattice sites. For this case, $P(s)$ and $\bar{P}(s)$ are drawn as the dotted line and the continuous line respectively in Fig. 2. From this figure it is evident that, the cumulative distribution of spacing matches with $\bar{P}(s)$ extremely well. The MSE for this case is obtained as $2.628 \times 10^{-6}$. Next, we consider the case of $s u(2 \mid 1)$ spin chain with $N=30$ lattice sites. The corresponding $P(s)$ and $\bar{P}(s)$ are plotted as the dotted line and the continuous line respectively in Fig. 3. Again, a very good agreement is found between these two lines with MSE given by $4.724 \times 10^{-4}$. By studying such particular cases we conclude that, similar to the case of other quantum integrable spin chains with long-range interaction that have been studied so far, the cumulative distribution of spacing between consecutive energy levels of the supersymmetric Polychronakos spin chain follows the expression (16) with remarkable accuracy. 


\section{References}

[1] F. Calogero, J. Math. Phys. 12 (1971) 419.

[2] B. Sutherland, Phys. Rev. A 4 (1971) 2019; B. Sutherland, Phys. Rev. A 5 (1972) 1372.

[3] M.A. Olshanetsky and A.M. Perelomov, Phys. Rep. 94 (1983) 313.

[4] F.D.M. Haldane, Phys. Rev. Lett. 60 (1988) 635.

[5] B. S. Shastry, Phys. Rev. Lett. 60 (1988) 639.

[6] N. Kawakami, Phys. Rev. B 46 (1992) 1005.

[7] Z.N.C. Ha and F.D.M. Haldane, Phys. Rev. B 46 (1992) 9359.

[8] A.P. Polychronakos, Phys. Rev. Lett. 70 (1993) 2329.

[9] A.P. Polychronakos, Nucl. Phys. B 419 (1994) 553.

[10] H. Frahm, J. Phys. A 26 (1993) L473.

[11] Z.N.C. Ha, Quantum many-body systems in one dimension, Series on Advances in Statistical Mechanics, Vol.12 (World Scientific,1996).

[12] M.V.N. Murthy and R. Shankar, Phys. Rev. Lett. 73 (1994) 3331.

[13] A.P. Polychronakos, Generalized statistics in one dimension, Les Houches 1998 lectures, hep-th/9902157.

[14] C.W.J. Beenakker and B. Rejaei, Phys. Rev. B 49 (1994) 7499; M. Caselle, Phys. Rev. Lett. 74 (1995) 2776.

[15] F. D. M. Haldane, Z.N.C. Ha, J.C. Talstra, D. Benard and V. Pasquier, Phys. Rev. Lett. 69 (1992) 2021. 
[16] D. Benard, M. Gaudin, F. D. M. Haldane, and V. Pasquier, J. Phys. A 26 (1993) 5219.

[17] K.Hikami, Nucl. Phys. B 441 (1995) 530.

[18] D. Berenstein, S.A. Cherkis, Nucl. Phys. B 702 (2004) 49.

[19] D. Serban, M. Staudacher, JHEP 0406 (2004) 001.

[20] N. Beisert, M. Staudacher, Nucl. Phys. B 727 (2005) 1.

[21] F. D. M Haldane, in Proc. 16th Taniguchi Symp., Kashikojima, Japan (1993), eds. A. Okiji and N. Kawakami (Springer, 1994).

[22] B. Basu-Mallick, Nucl. Phys. B 540 (1999) 679.

[23] B. Basu-Mallick, H. Ujino and M. Wadati, Jour. Phys. Soc. Jpn. 68 (1999) 3219.

[24] K. Hikami and B. Basu-Mallick, Nucl. Phys. B 566 (2000) 511.

[25] B. Basu-Mallick and N. Bondyopadhaya, Nucl. Phys. B 757 (2006) 280.

[26] B. Basu-Mallick, N. Bondyopadhaya and D. Sen, Nucl. Phys. B 795 (2008) 596.

[27] R. Thomale, D. Schuricht and M. Greiter, Phys. Rev. B 74 (2006) 024423.

[28] P. Schlottmann, Int. Jour. Mod. Phys. B 11 (1997) 355.

[29] M. Arikawa, Y. Saiga and Y. Kuramoto, Phys. Rev. Lett. 86 (2001) 3096; M. Arikawa and Y. Saiga, J. Phys. A 39 (2006) 10603.

[30] D. Bernard, V. Pasquier and D. Serban, Europhys. Lett. 30 (1995) 301.

[31] T. Yamamoto and O. Tsuchiya, J. Phys. A 29 (1996) 3977.

[32] E. Corrigan and R. Sasaki, J. Phys. A 35 (2002) 7017. 
[33] A. Enciso, F. Finkel, A. González-López and M.A. Rodríguez, Nucl. Phys. B 707 (2005) 553.

[34] J. C. Barba, F. Finkel, A. González-López and M.A. Rodríguez, Phys. Rev. B 77 (2008) 214422.

[35] J. C. Barba, F. Finkel, A. González-López and M.A. Rodríguez, Nucl. Phys. B 806 (2009) 684 .

[36] B. Basu-Mallick, F. Finkel and A. González-López, arXiv: 0809.4234, to be published in Nuclear Physics B.

[37] F. Finkel and A. González-López, Phys. Rev. B 72 (2005) 174411.

[38] J. C. Barba, F. Finkel, A. González-López and M.A. Rodríguez, Europhys Lett. 83 (2008) 27005.

[39] M. V. Berry and M. Tabor, Proc. R. Soc. Lond. A 356 (1977), 375

[40] S. Ahmed, Lett. Nuovo Cimento 22 (1978) 367.

[41] F. Haake, Quantum signatures of Chaos (Springer-verlag, 2001). 


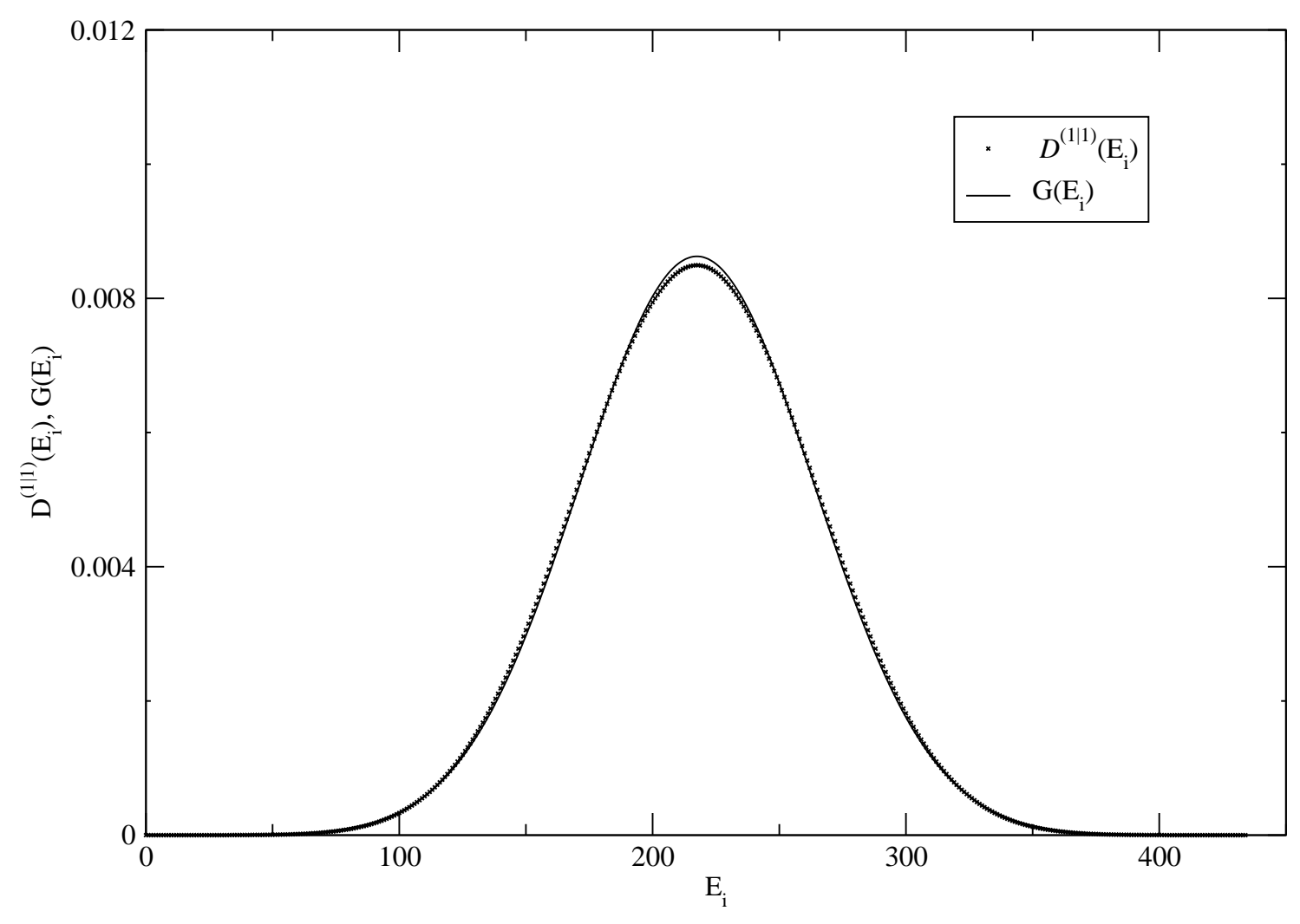

Figure 1: Plot of the degeneracies $\mathcal{D}^{(1 \mid 1)}\left(E_{i}\right)$ versus energies $E_{i}$ (dotted line) for the case of $s u(1 \mid 1)$ Polychronakos spin chain with $N=30$, and its comparison with the Gaussian distribution (continuous line). 


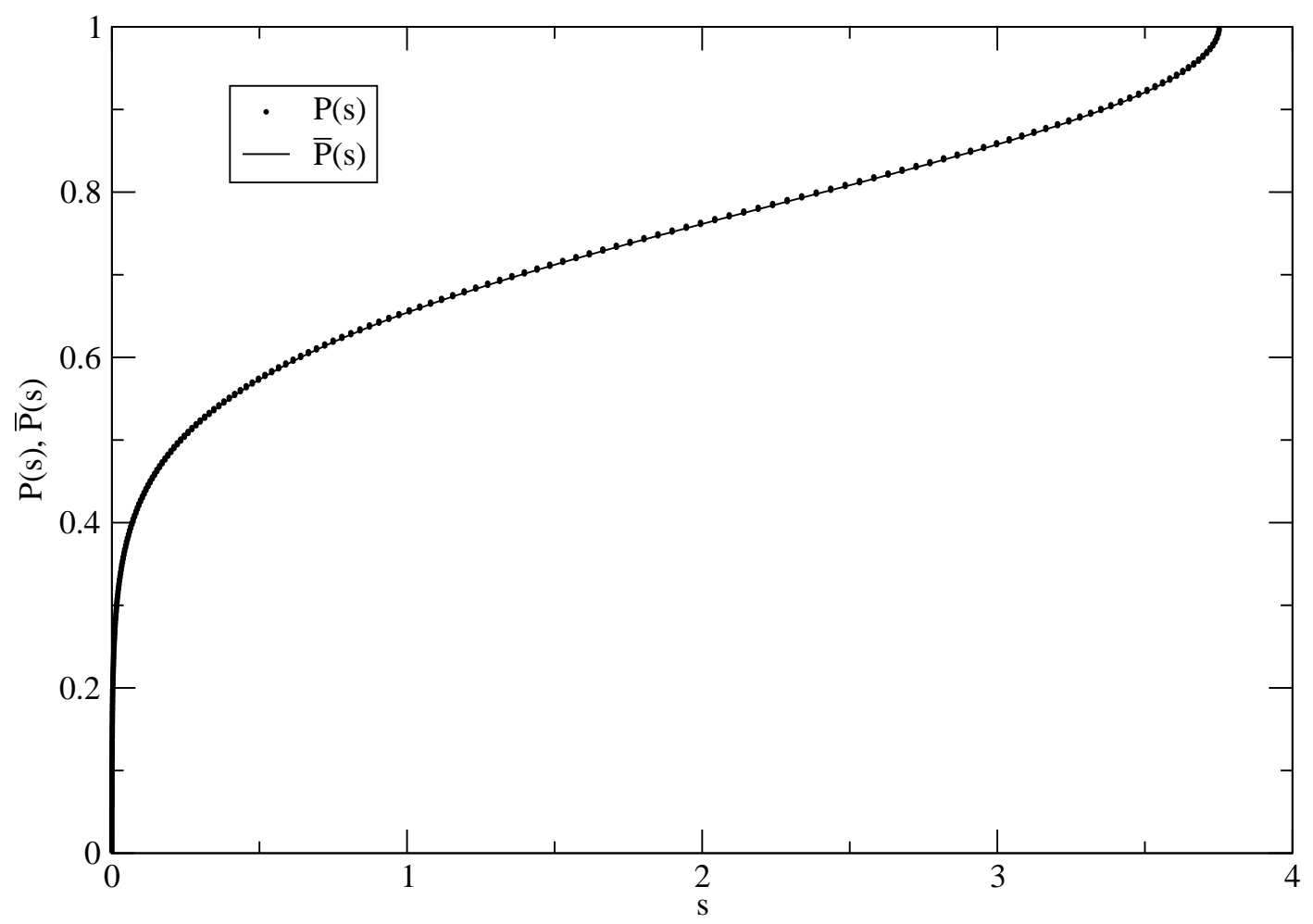

Figure 2: The dotted curve represents the cumulative distribution of spacing between consecutive energy levels for $s u(1 \mid 1)$ spin chain with $N=30$, and the continuous curve represents the corresponding $\bar{P}(s)$. 


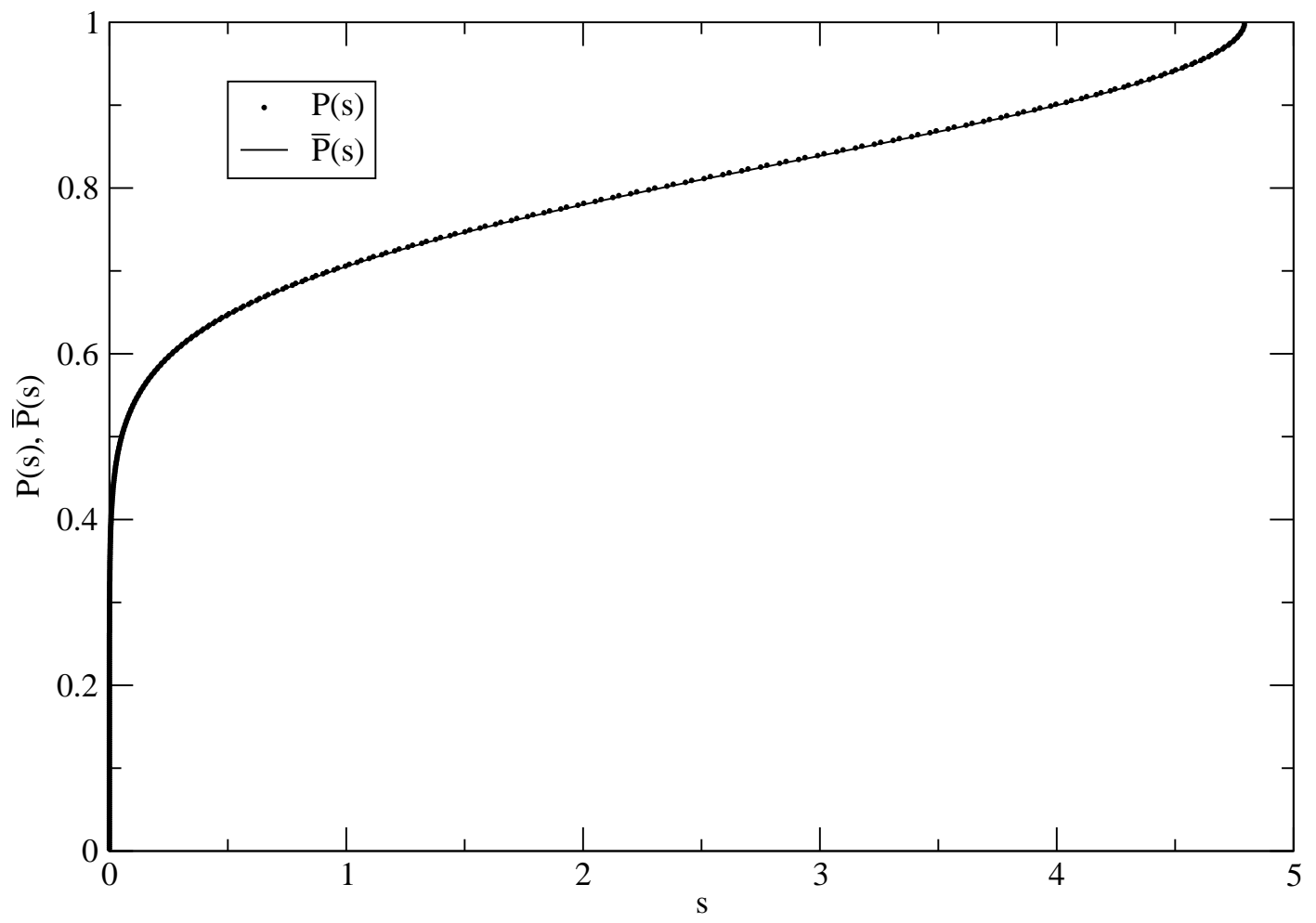

Figure 3: The dotted curve represents the cumulative distribution of spacing between consecutive energy levels for $s u(2 \mid 1)$ spin chain with $N=30$, and the continuous curve represents the corresponding $\bar{P}(s)$. 